\title{
Survey of Tephritidae and Lonchaeidae (Diptera), their host plants and parasitoids in the state of Sergipe, Brazil
}

\author{
Marliton Rocha BARRETO ${ }^{1 *}$, Ricardo ADAIME2 2 , Maria do Socorro Miranda de SOUSA3, \\ Miguel Francisco de SOUZA-FILHO 4 , Pedro Carlos STRIKIS 5 , Adenir Vieira TEODORO ${ }^{6}$, \\ Roberto Antonio $\mathrm{ZUCCHI}^{7}$
}

\author{
${ }^{1}$ Instituto de Ciências Naturais, Humanas e Sociais, Universidade Federal de Mato Grosso, Sinop, MT, Brasil. \\ ${ }^{2}$ Centro de Pesquisa Agroflorestal do Amapá, Embrapa, Macapá, AP, Brasil. (ORCID: 0000-0001-8044-3976) \\ 3 Programa de Pós-Graduação em Biodiversidade Tropical, Universidade Federal do Amapá, Macapá, AP, Brasil. \\ (ORCID: 0000-0002-0227-7340) \\ ${ }^{4}$ Centro Avançado de Pesquisa em Proteção de Plantas e Saúde Animal, Instituto Biológico, Campinas, SP, Brasil. \\ (ORCID: 0000-0001-7838-1489) \\ ${ }^{5}$ Instituto de Geociências, Universidade de São Paulo, SP, Brasil. (ORCID: 0000-0001-6249-2344) \\ ${ }^{6}$ Centro de Pesquisa Agropecuária dos Tabuleiros Costeiros, Embrapa, Aracajú, SE, Brasil. (ORCID: 0000-0001-9490-0832) \\ ${ }^{7}$ Escola Superior de Agricultura Luiz de Queiroz, Universidade de São Paulo, Piracicaba, SP, Brasil. (ORCID: 0000-0001-9861-7460) \\ *E-mail: mrb.ufmt@gmail.com (ORCID: 0000-0003-3793-8855)
}

Recebido em 05/07/2019; Aceito em 14/05/2020; Publicado em 09/06/2020.

\begin{abstract}
This is the first survey of Tephritidae and Lonchaeidae, their host plants and parasitoids in the state of Sergipe, Brazil. Fruits of several plant species were sampled in orchards, backyards, and farmers markets of 13 municipalities, from October 22 to 26, 2018 and from January 7 to 11, 2019. We collected 89 fruit samples (4,930 fruits, $111.5 \mathrm{~kg}$ ) from 28 plant species belonging to 17 families. Larval infestation occurred in 29 fruit samples belonging to 11 plant species from eight families. We also used McPhail traps baited with Ceratrap ${ }^{\circledR}$, in five municipalities. Five species of fruit flies (Anastrepha spp. and Ceratitis capitata), three species of lance flies (Neosilba spp.), nine of host plants, and associated parasitoids (1 species) were recorded for the first time in the state of Sergipe.
\end{abstract}

Key words: Anastrepha; Ceratitis capitata; Neosilba; Doryctobracon areolatus; fruit flies; lance flies; trap.

\section{Levantamento de Tephritidae e Lonchaeidae (Diptera), suas plantas hospedeiras e parasitoide no estado de Sergipe, Brasil}

\begin{abstract}
RESUMO: Este é o primeiro levantamento de espécies de Tephritidae e Lonchaeidae, suas plantas hospedeiras e parasitoides no estado de Sergipe, Brasil. Foram amostrados frutos de diversas espécies em pomares, quintais e feiras livres de 13 municípios, de 22 a 26 de outubro de 2018 e de 7 a 11 de janeiro de 2019. Foram coletadas 89 amostras de frutos (4.930 frutos; $111,5 \mathrm{~kg}$ ) de 28 espécies vegetais pertencentes a 17 famílias. Houve infestação de larvas em 29 amostras de frutos pertencentes a 11 espécies vegetais de 8 famílias. Também foram usadas armadilhas tipo McPhail com o atrativo Ceratrap ${ }^{\circledR}$, em cinco municípios. Cinco espécies de tefritídeos (Anastrepha spp. e Ceratitis capitata), três de lonqueídeos (Neosilba spp.), nove de hospedeiros e uma de parasitoides são registradas pela primeira vez no estado de Sergipe.

Palavras-chave: Anastrepha; Ceratitis capitata; Neosilba; Doryctobracon areolatus; moscas-das-frutas; lonqueídeos; armadilha.
\end{abstract}

\section{INTRODUCTION}

The state of Sergipe is the second major producer of orange and lemon in northeastern Brazil and the state produces almost all the orange juice of the region. Nevertheless, despite the yield of orange ( $3^{\text {rd }}$ place of average yield/ha) and several native fruits, studies on dipterans associated to fruits have been neglected in Sergipe.

Several species of fruit flies (Tephritidae) are important pests worldwide. In Brazil, these dipterans are represented by native species of Anastrepha and two introduced species Ceratitis capitata (Mediterranean fruit fly) and Bactrocera carambolae (carambola fruit fly). Species of Anastrepha are pests - A. fraterculus (lato sensu), A. obliqua, A. sororcula, A. grandis and $A$. pseudoparallela - and occur in several Brazilian states (e.g. MALAVASI; ZUCCHI, 2000). The Mediterranean fruit fly, introduced at the beginning of the $20^{\text {th }}$ century, is widely distributed in Brazil (ZUCCHI, 2015). The carambola fruit fly, detected in Brazil in 1996, has a restricted distribution to states of Amapá, Pará and Roraima, kept under official control by the Ministry of Agriculture, Livestock and Supply (BRASIL, 2018).

Some species of Lonchaeidae may damage fruits and other structures of some fruit trees (UCHÔA, 2012; ADAIME et al., 2017). However, damages caused by lance flies have less economic importance than those caused by Tephritidae, as they attack few species of fruit trees and are not considered quarantine species. Damages caused by Tephritidae and lance flies are caused by larvae, which 
develop inside the fruits, making them unfit for consumption and commercialization.

In the Americas, Brazil holds most studies on diversity of fruit flies; however, no studies on fruit flies or lance flies have been conducted in the state of Sergipe. Therefore, this study presents the results of the first survey on dipterans in fruits and traps in the state of Sergipe, Brazil.

\section{MATERIALS AND METHODS}

\subsection{Sampling sites}

Fruits were sampled in 13 municipalities (Aracaju, Areia Branca, Barra dos Coqueiros, Canindé de São Francisco, Itabaiana, Itaporanga D’Ajuda, Lagarto, Poço Redondo, Ribeirópolis, Salgado, São Cristóvão, Siriri and Tobias Barreto) (Table 1). However, collections with traps were carried out in only five municipalities (Table 2).

Sergipe has high average temperatures and small annual temperature variation, presenting average range below $5^{\circ} \mathrm{C}$ and rainfall decreasing from the coast to the inner part of the state. Sergipe has three climatic zones: Tropical humid, along the coast (East); Tropical sub-humid or Transitional SemiArid (Agreste) and Hinterland (Sertão) (SANTOS, 2019; SEMARH, 2019). During the collections in January the average temperature was $26.5^{\circ} \mathrm{C}$, with an average precipitation of $49 \mathrm{~mm}$ (Litoral and Agreste) and $27.3^{\circ} \mathrm{C}$ and $38 \mathrm{~mm}$ (Sertão). In October, the average temperature was $25.4^{\circ} \mathrm{C}$, with an average rainfall of $56 \mathrm{~mm}$ (Litoral and Agreste) and $27.3^{\circ} \mathrm{C}$ and $9 \mathrm{~mm}$ in the Sertão.

\subsection{Fruit sampling}

Fruits potentially infested by frugivorous larvae were collected in orchards and backyards, from October 22 to 26, 2018 and from January 7 to 11, 2019. Samples were collected randomly, and newly-dropped fruits were collected in the soil and/or directly from the plants sampled. All sampling sites had their geographical coordinates registered by Global Positioning System (GPS) (Table 1). In municipalities of Barra dos Coqueiros, Lagarto, Ribeirópolis, Tobias Barreto and Salgado, the fruits were purchased at farmers markets.

Fruits of the same species were grouped and packed in plastic containers, wrapped with organza bag, duly labeled, and transported to the Laboratory of Entomology at Embrapa Tabuleiros Costeiros in Aracaju. In the laboratory, fruits were counted, weighed and arranged in plastic trays on a thin layer of moistened sand. The trays were covered with organza fabric fastened by elastic alloy (SILVA et al., 2011).

Fruits were examined every five days for 20 days to remove the puparia, which were transferred to transparent plastic bottles containing a thin layer of moist vermiculite. The bottles were covered with organza fabric with the lids punctured, and inspected daily until insect emergence. Humidity in trays and pots was kept by replacing water, using a pissette. Emerged insects (flies and parasitoids) were kept alive for 24 hours to fix the wing patterns, and after killed, they were placed into micro tubes containing $70 \%$ ethanol, duly labeled, for later identification. The scientific names of plant species are in accordance with The Plant List (2013) and Flora do Brasil (2020).

\subsection{Sampling with traps}

We used $10 \mathrm{McPhail}$ traps of transparent plastic with bottom opening, containing $400 \mathrm{~mL}$ of bait Ceratrap ${ }^{\circledR}$, installed $1.8 \mathrm{~m}$ above the ground. All sampling sites where the traps were installed had their geographical coordinates recorded by GPS (Table 2). The traps were installed on $10 / 22 / 18$ and $01 / 07 / 19$ and were checked only once on $10 / 26 / 18$ and $01 / 11 / 19$, respectively, when the insects were transferred to flasks containing $70 \%$ ethanol and dully labeled.

\subsection{Fly identification}

Identification of flies and parasitoids was based exclusively on adults. Specific identification of Anastrepha was based on females (aculeus apex) and C. capitata on males and females (external morphological features) (MF Souza-Filho). Identification of lance flies was based on males (PC Strikis). Identification of braconids was based on Marinho et al. (2018). Anastrepha fraterculus corresponds to a complex of cryptic species; however, considered as lato sensu in this paper. Voucher specimens are deposited at the Plant Protection Laboratory at Embrapa Amapá.

\subsection{Data analyses}

The following calculations were performed for each fruit sample: (1) infestation index [number of puparia divided by sample weight (puparia/kg of fruit)] and (2) parasitism rate (number of parasitoids divided by number of puparia, multiplied by 100).

Table 1. Fruit collection sites in municipalities of Sergipe.

Tabela 1. Pontos de coleta de frutos em municípios de Sergipe.

\begin{tabular}{|c|c|c|c|}
\hline Spots* & Municipalities & Latitude S & Longitude W \\
\hline 1 & São Cristóvão & $10^{\circ} 58^{\prime} 18.0^{\prime \prime}$ & $37^{\circ} 10^{\prime} 43.1^{\prime \prime}$ \\
\hline 2 & São Cristóvão & $10^{\circ} 58^{\prime} 19.8^{\prime \prime}$ & $37^{\circ} 12^{\prime} 59.9^{\prime \prime}$ \\
\hline 3 & Itaporanga D'Ajuda & $10^{\circ} 59^{\prime} 45.2^{\prime \prime}$ & $37^{\circ} 20^{\prime} 0.03^{\prime \prime}$ \\
\hline 4 & Itaporanga D'Ajuda & $10^{\circ} 58^{\prime} 49.3^{\prime \prime}$ & $37^{\circ} 24^{\prime} 01.1^{\prime \prime}$ \\
\hline 5 & Itaporanga D'Ajuda & $10^{\circ} 58^{\prime} 20.3^{\prime \prime}$ & $37^{\circ} 27^{\prime} 01.9^{\prime \prime}$ \\
\hline 6 & Itaporanga D'Ajuda & $10^{\circ} 56^{\prime} 44.3^{\prime \prime}$ & $37^{\circ} 28^{\prime} 45.3^{\prime \prime}$ \\
\hline 7 & Areia Branca & $10^{\circ} 45^{\prime} 41.6^{\prime \prime}$ & $37^{\circ} 18^{\prime} 05.4^{\prime \prime}$ \\
\hline 8 & Areia Branca & $10^{\circ} 45^{\prime} 38.2^{\prime \prime}$ & $37^{\circ} 18^{\prime} 20.8^{\prime \prime}$ \\
\hline 9 & Areia Branca & $10^{\circ} 46^{\prime} 11.3^{\prime \prime}$ & $37^{\circ} 22^{\prime} 05.6^{\prime \prime}$ \\
\hline 10 & Itabaiana & $10^{\circ} 44^{\prime} 17.3^{\prime \prime}$ & $37^{\circ} 24^{\prime} 22.2^{\prime \prime}$ \\
\hline 11 & Poço Redondo & $09^{\circ} 42^{\prime} 59.2^{\prime \prime}$ & $37^{\circ} 45^{\prime} 48.6^{\prime \prime}$ \\
\hline 12 & Poço Redondo & $09^{\circ} 42^{\prime} 37.3^{\prime \prime}$ & $37^{\circ} 46^{\prime} 12.7^{\prime \prime}$ \\
\hline 13 & C. de São Francisco & $09^{\circ} 42^{\prime} 31.6^{\prime \prime}$ & $37^{\circ} 46^{\prime} 09.7^{\prime \prime}$ \\
\hline 14 & C. de São Francisco & $09^{\circ} 40^{\prime} 58.7^{\prime \prime}$ & $37^{\circ} 48^{\prime} 43.8^{\prime \prime}$ \\
\hline 15 & C. de São Francisco & $09^{\circ} 40^{\prime} 16.5^{\prime \prime}$ & $37^{\circ} 49^{\prime} 04.2^{\prime \prime}$ \\
\hline 16 & C. de São Francisco & $09^{\circ} 40^{\prime} 01.3^{\prime \prime}$ & $37^{\circ} 49^{\prime} 11.3^{\prime \prime}$ \\
\hline 17 & C. de São Francisco & $09^{\circ} 41^{\prime} 10.6^{\prime \prime}$ & $37^{\circ} 49^{\prime} 01.0^{\prime \prime}$ \\
\hline 18 & C. de São Francisco & $09^{\circ} 40^{\prime} 49.3^{\prime \prime}$ & $37^{\circ} 48^{\prime} 28.4^{\prime \prime}$ \\
\hline 19 & Siriri & $10^{\circ} 34^{\prime} 36.9^{\prime \prime}$ & $37^{\circ} 07^{\prime} 36.4^{\prime \prime}$ \\
\hline 20 & Itaporanga D'Ajuda & $10^{\circ} 58^{\prime} 49.3^{\prime \prime}$ & $37^{\circ} 24^{\prime} 01.1^{\prime \prime}$ \\
\hline 21 & Itaporanga D'Ajuda & $11^{\circ} 12^{\prime} 50.4^{\prime \prime}$ & $37^{\circ} 13^{\prime} 01.2^{\prime \prime}$ \\
\hline 22 & Aracaju & $10^{\circ} 59^{\prime} 58.7^{\prime \prime}$ & $37^{\circ} 03^{\prime} 24.3^{\prime \prime}$ \\
\hline 23 & Areia Branca & $10^{\circ} 45^{\prime} 45.9^{\prime \prime}$ & $37^{\circ} 18^{\prime} 08.5^{\prime \prime}$ \\
\hline 24 & Itabaiana & $10^{\circ} 43^{\prime} 32.5^{\prime \prime}$ & $37^{\circ} 23^{\prime} 48.8^{\prime \prime}$ \\
\hline 25 & Itaporanga D'Ajuda & $10^{\circ} 59^{\prime} 50.0^{\prime \prime}$ & $37^{\circ} 19^{\prime} 52.0^{\prime \prime}$ \\
\hline 26 & São Cristóvão & $10^{\circ} 59^{\prime} 23.9^{\prime \prime}$ & $37^{\circ} 13^{\prime} 05.2^{\prime \prime}$ \\
\hline 27 & São Cristóvão & $11^{\circ} 00^{\prime} 12.6^{\prime \prime}$ & $37^{\circ} 12^{\prime} 36.2^{\prime \prime}$ \\
\hline 28 & Aracaju & $10^{\circ} 57^{\prime} 06.1 "$ & $37^{\circ} 03^{\prime} 14.9^{\prime \prime}$ \\
\hline 29 & Aracaju & $10^{\circ} 56^{\prime} 06.4^{\prime \prime}$ & $37^{\circ} 04^{\prime} 45.6^{\prime \prime}$ \\
\hline
\end{tabular}

${ }^{*}$ Fruits from municipalities of Barra dos Coqueiros, Lagarto, Ribeirópolis, Tobias Barreto, and Salgado were purchased at farmers markets and were not included in this table. 


\section{Barreto et al.}

Table 2. Collection sites with McPhail traps in municipalities of Sergipe (January 7 to 11, 2019).

Tabela 2. Pontos de coleta com armadilhas tipo McPhail em municípios de Sergipe (7 a 11 de janeiro de 2019).

\begin{tabular}{lcc}
\hline Municipalities & Latitude S & Longitude W \\
\hline \multirow{2}{*}{ Aracaju } & $10^{\circ} 57^{\prime} 06.1^{\prime \prime}$ & $37^{\circ} 03^{\prime} 14.9^{\prime \prime}$ \\
& $11^{\circ} 04^{\prime} 19.3^{\prime \prime}$ & $37^{\circ} 07^{\prime} 20.3^{\prime \prime}$ \\
\hline Areia Branca* & $10^{\circ} 45^{\prime} 41.6^{\prime \prime}$ & $37^{\circ} 18^{\prime} 05.4^{\prime \prime}$ \\
\hline \multirow{2}{*}{ Itabaiana } & $10^{\circ} 44^{\prime} 17.3^{\prime \prime}$ & $37^{\circ} 24^{\prime} 22.2^{\prime \prime}$ \\
& $10^{\circ} 43^{\prime} 32.5^{\prime \prime}$ & $37^{\circ} 23^{\prime} 48.8^{\prime \prime}$ \\
\hline \multirow{2}{*}{ Itaporanga D'Ajuda* } & $10^{\circ} 58^{\prime} 49.3^{\prime \prime}$ & $37^{\circ} 24^{\prime} 01.1^{\prime \prime}$ \\
& $10^{\circ} 58^{\prime} 20.3^{\prime \prime}$ & $37^{\circ} 27^{\prime} 01.9^{\prime \prime}$ \\
& $10^{\circ} 56^{\prime} 44.3^{\prime \prime}$ & $37^{\circ} 28^{\prime} 45.3^{\prime \prime}$ \\
& $11^{\circ} 12^{\prime} 50.4^{\prime \prime}$ & $37^{\circ} 13^{\prime} 01.2^{\prime \prime}$ \\
& $10^{\circ} 59^{\prime} 50.0^{\prime \prime}$ & $37^{\circ} 19^{\prime} 52.0^{\prime \prime}$ \\
São Cristóvão & $10^{\circ} 59^{\prime} 45.2^{\prime \prime}$ & $37^{\circ} 20^{\prime} 0.03^{\prime \prime}$ \\
\hline
\end{tabular}

*Sampling carried out from Oct 23-26, 2018.

\section{RESULTS}

We collected 89 fruit samples (4,930 fruits, $111.5 \mathrm{~kg})$ from 28 plant species belonging to 17 families (Table 3) with the highest number of samples of Malpighia emarginata (12 samples) and Mangifera indica (11 samples).

Infestation occurred in 29 samples $(32.6 \%)$, belonging to 11 plant species from eight families (Table 3). Infestation rates varied, with the highest index recorded in a sample of Manihot esculenta (366.7 puparia/ $\mathrm{kg}$ of fruit) in the municipality of Areia Branca (Table 3).

We obtained 1,153 puparia, from which we observed emergence of five species of Tephritidae ( $A$. fraterculus, $A$. obliqua, $A$. pickeli, $A$. sororcula and Ceratitis capitata) and three species of lance flies (Neosilba certa, N. inesperata and $N$. pendula) and one parasitoid species (Doryctobracon areolatus) (Table 3).

Ceratitis capitata was associated to more hosts (6 species in 6 families) than the other species of flies collected. Among species of Anastrepha, A. obliqua was associated with four host plants ( 2 families), $A$. fraterculus in two host families, and $A$. pickeli and $A$. sororcula infested only one host (Table 3). Ceratitis capitata and $A$. obliqua were the most widely distributed species occurring in six municipalities (Figure 1). Anastrepha fraterculus and $A$. pickeli occurred in four municipalities, $A$. sororcula in three and $A$. psendoparallela in only one (Figure 1).

From fruits sampled in farmers markets, A. obliqua was obtained in Spondias purpurea (São Cristóvão) and C. capitata and $A$. sororcula in Psidium guajava (Canindé de São Francisco) (Table 3).

Three species of Neosilba $-N$. certa, $N$. inesperata and $N$. pendula - infested Malpighia emarginata; however, $N$. certa also infested Citrus reticulata (Table 3).

In the traps, we captured specimens of Anastrepha obliqua (292 +), A. fraterculus (9 +), A. pickeli (7 +), A. sororcula (4 9), A. pseudoparallela ( $3 q$ and $60 \lambda$ ), C. capitata $(43 q$ and $\left.9 \delta^{\lambda}\right)$ and Neosilba sp. (1 9 ) (Table 4). Ceratitis capitata and $A$. obliqua were collected in more municipalities (5 and 4, respectively) than the other species sampled by traps (Table 4).

Doryctobracon areolatus (Szépligeti) (Hymenoptera: Braconidae) was the only parasitoid collected. Only eight specimens of the parasitoid were collected associated to $A$. obliqua in Averrboa carambola (Areia Branca and São Cristóvão) and in Spondias dulcis (Itaporanga D'Ajuda). Parasitism rates were $2.8 \%$ in $S$. dulcis in the municipality of Itaporanga D'Ajuda and $5.1 \%$ and $4.5 \%$ in $A$. carambola, in the municipalities of Areia Branca and São Cristóvão, respectively.

Table 3. Flies (Tephritidae and Lonchaeidae) and their hosts in municipalities of the state of Sergipe. October 2018 and January 2019.

Tabela 3. Moscas (Tephritidae e Lonchaeidae) e respectivos hospedeiros em municípios do estado de Sergipe. Outubro de 2018 e janeiro de 2019

\begin{tabular}{|c|c|c|c|c|c|c|c|}
\hline $\begin{array}{l}\text { Families* } \\
\text { Species }\end{array}$ & Spots ${ }^{\#}$ & Flies / Parasitoids & Dates & $\mathrm{F}(\mathrm{n})$ & M (kg) & $\mathrm{PP}(\mathrm{n})$ & $\mathrm{I}(\mathrm{PP} / \mathrm{kg})$ \\
\hline \multicolumn{8}{|l|}{ Anacardiaceae } \\
\hline Anacardium bumile A.St.-Hil. & $\mathrm{R}^{* *}$ & - & 01/08/19 & 10 & 0.32 & 0 & 0 \\
\hline \multirow[t]{2}{*}{ Anacardium occidentale $\mathrm{L}$. } & $\mathrm{SC}^{1}$ & - & $10 / 22 / 18$ & 4 & 0.52 & 0 & 0 \\
\hline & $\mathrm{SC}^{26}$ & - & 01/07/19 & 12 & 0.72 & 0 & 0 \\
\hline \multirow[t]{11}{*}{ Mangifera indica $\mathrm{L}$. } & $\mathrm{SC}^{1}$ & Ao $(2+), 2 \hat{\jmath}$ & $10 / 22 / 18$ & 14 & 1.36 & 6 & 4.4 \\
\hline & $\mathrm{AB}^{9}$ & Ao $(1+), 2{ }^{\Uparrow} \mathrm{A}$ & $10 / 23 / 18$ & 13 & 1.30 & 3 & 2.3 \\
\hline & $\mathrm{PR}^{11}$ & - & $10 / 24 / 18$ & 8 & 1.30 & 0 & 0 \\
\hline & $\mathrm{CSF}^{14}$ & - & $10 / 24 / 18$ & 3 & 1.85 & 0 & 0 \\
\hline & $\mathrm{CSF}^{17}$ & - & $10 / 24 / 18$ & 9 & 3.60 & 0 & 0 \\
\hline & $\mathrm{CSF}^{18}$ & - & $10 / 24 / 18$ & $11^{\mathrm{a}}$ & 4.85 & 0 & 0 \\
\hline & $\mathrm{S}^{19}$ & Ao $(1+$ ) $), 2 \hat{\jmath} \mathrm{A}$ & $10 / 25 / 18$ & $12^{\mathrm{b}}$ & 2.90 & 4 & 1.4 \\
\hline & $\mathrm{I}^{10}$ & - & 01/07/19 & $10^{\mathrm{a}}$ & 1.56 & 0 & 0 \\
\hline & $\mathrm{I}^{24}$ & - & 01/07/19 & $9^{c}$ & 1.56 & 0 & 0 \\
\hline & $\mathrm{ID}^{* *}$ & - & 01/07/19 & $12^{c}$ & 3.36 & 0 & 0 \\
\hline & $\mathrm{SC}^{1}$ & $2 \lesssim \mathrm{A}$ & $01 / 07 / 19$ & $15^{\mathrm{a}}$ & 4.50 & 6 & 1.3 \\
\hline \multirow[t]{3}{*}{ Spondias dulcis Parkinson } & $\mathrm{SC}^{1}$ & - & $10 / 22 / 18$ & 3 & 0.16 & 0 & 0 \\
\hline & $\mathrm{SC}^{1}$ & - & $10 / 26 / 18$ & 5 & 0.16 & 0 & 0 \\
\hline & $\mathrm{ID}^{25}$ & Ao $(3+), 7 \hat{A}, \mathrm{Da}(1)$ & 01/07/19 & 37 & 1.30 & 36 & 27.7 \\
\hline \multirow[t]{5}{*}{ Spondias purpurea L. } & $\mathrm{PR}^{12}$ & - & $10 / 24 / 18$ & 255 & 1.70 & 0 & 0 \\
\hline & $\mathrm{CSF}^{14}$ & - & $10 / 24 / 18$ & 56 & 1.30 & 0 & 0 \\
\hline & $\mathrm{CSF}^{17}$ & Ao (1우), $1 \jmath^{A}$ & $10 / 24 / 18$ & 172 & 3.70 & 6 & 1.6 \\
\hline & $\mathrm{SC}^{* *}$ & Ao (109), $6 \bigcirc^{A} \mathrm{~A}$ & $01 / 10 / 19$ & 83 & 0.69 & 28 & 40.6 \\
\hline & $\mathrm{A}^{29}$ & 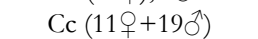 & $01 / 10 / 19$ & 54 & 0.30 & 32 & 106.7 \\
\hline
\end{tabular}


Table 3. Flies (Tephritidae and Lonchaeidae) and their hosts in municipalities of the state of Sergipe. Continuation...

Tabela 3. Moscas (Tephritidae e Lonchaeidae) e respectivos hospedeiros em municípios do estado de Sergipe. Continuação...

\begin{tabular}{|c|c|c|c|c|c|c|c|}
\hline $\begin{array}{l}\text { Families* } \\
\text { Species }\end{array}$ & Spots ${ }^{\#}$ & Flies / Parasitoids & Dates & $\mathrm{F}(\mathrm{n})$ & M (kg) & $\mathrm{PP}(\mathrm{n})$ & $\mathrm{I}(\mathrm{PP} / \mathrm{kg})$ \\
\hline \multicolumn{8}{|l|}{ Annonaceae } \\
\hline \multirow[t]{4}{*}{ Annona muricata $\mathrm{L}$. } & $\mathrm{SC}^{2}$ & - & $10 / 22 / 18$ & 1 & 1.86 & 0 & 0 \\
\hline & $\mathrm{ID}^{4}$ & - & $10 / 22 / 18$ & 2 & 1.56 & 0 & 0 \\
\hline & $\mathrm{AB}^{7}$ & - & $10 / 23 / 18$ & 1 & 1.04 & 0 & 0 \\
\hline & $\mathrm{CSF}^{17}$ & - & $10 / 24 / 18$ & 3 & 2.10 & 0 & 0 \\
\hline Xylopia sp. & $\mathrm{ID}^{3}$ & - & $10 / 22 / 18$ & 149 & 0.08 & 0 & 0 \\
\hline \multicolumn{8}{|l|}{ Apocynaceae } \\
\hline \multirow{2}{*}{ Hancornia speciosa Gomes } & $\mathrm{BC}^{* *}$ & - & $10 / 25 / 18$ & 54 & 0.58 & 0 & 0 \\
\hline & $\mathrm{ID}^{21}$ & - & $10 / 26 / 18$ & 71 & 1.68 & 0 & 0 \\
\hline \multicolumn{8}{|l|}{ Fabaceae } \\
\hline Tamarindus indica $\mathrm{L}$. & $\mathrm{ID}^{4}$ & - & $10 / 22 / 18$ & 10 & 0.46 & 0 & 0 \\
\hline \multicolumn{8}{|l|}{ Combretaceae } \\
\hline \multirow[t]{4}{*}{ Terminalia catappa $\mathrm{L}$. } & $\mathrm{AB}^{7}$ & - & $10 / 23 / 18$ & 19 & 0.48 & 0 & 0 \\
\hline & $\mathrm{A}^{22}$ & $\mathrm{Cc}(34+9+49 \widehat{\jmath}) ; \operatorname{Af}(19), 2 \widehat{A}$ & $01 / 07 / 19$ & 43 & 1.14 & 132 & 115.8 \\
\hline & $\mathrm{SC}^{27}$ & $-x^{2}+100$ & $01 / 07 / 19$ & 103 & 1.50 & 0 & 0 \\
\hline & $\mathrm{A}^{28}$ & - & $01 / 07 / 19$ & 30 & 1.24 & 0 & 0 \\
\hline \multicolumn{8}{|l|}{ Euphorbiaceae } \\
\hline \multirow[t]{3}{*}{ Manibot esculenta Crantz } & $\mathrm{SC}^{1}$ & Ap (7우), $8 \widehat{\jmath} \mathrm{A}$ & $10 / 22 / 18$ & 117 & 0.24 & 27 & 112.5 \\
\hline & $\mathrm{AB}^{7}$ & Ap (6ㅇ), $9 \widehat{A}$ & $10 / 23 / 18$ & 22 & 0.06 & 22 & 366.7 \\
\hline & $\mathrm{S}^{19}$ & 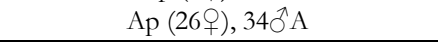 & $10 / 25 / 18$ & 209 & 0.85 & 90 & 105.9 \\
\hline \multicolumn{8}{|l|}{ Lythraceae } \\
\hline \multirow[t]{2}{*}{ Punica granatum $\mathrm{L}$. } & $I^{10}$ & - & $10 / 23 / 18$ & 6 & 0.64 & 0 & 0 \\
\hline & $\mathrm{CSF}^{14}$ & - & $10 / 24 / 18$ & 7 & 4.00 & 0 & 0 \\
\hline \multicolumn{8}{|l|}{ Malpighiaceae } \\
\hline Bunchosia armeniaca (Cav.) DC. & $\mathrm{AB}^{23}$ & - & $01 / 07 / 19$ & 56 & 0.30 & 0 & 0 \\
\hline \multirow[t]{2}{*}{ Byrsonima crassifolia (L.) Kunth } & $\mathrm{BC}^{* *}$ & - & $01 / 08 / 19$ & - & 0.30 & 0 & 0 \\
\hline & $\mathrm{BC}^{* *}$ & - & 01/08/19 & - & 0.30 & 0 & 0 \\
\hline \multirow[t]{12}{*}{ Malpigbia emarginata DC. } & $\mathrm{SC}^{1}$ & - & $10 / 22 / 18$ & 8 & 0.06 & 0 & 0 \\
\hline & $\mathrm{ID}^{3}$ & - & $10 / 22 / 18$ & 39 & 0.10 & 0 & 0 \\
\hline & $\mathrm{ID}^{4}$ & $\mathrm{Cc}\left(2 q+2 \sigma^{\pi}\right)$ & $10 / 22 / 18$ & 82 & 0.62 & 7 & 11.3 \\
\hline & $\mathrm{AB}^{7}$ & - & $10 / 23 / 18$ & 31 & 0.20 & 0 & 0 \\
\hline & $\mathrm{AB}^{8}$ & - & $10 / 23 / 18$ & 36 & 0.32 & 0 & 0 \\
\hline & $\mathrm{AB}^{8}$ & - & $10 / 23 / 18$ & 126 & 0.62 & 0 & 0 \\
\hline & $\mathrm{CSF}^{13}$ & $\mathrm{Ni}(1 \overbrace{}^{\jmath})$ & $10 / 24 / 18$ & 298 & 1.36 & 1 & 0,7 \\
\hline & $\operatorname{CSF}^{15}$ & - & $10 / 24 / 18$ & 125 & 1.10 & 0 & 0 \\
\hline & $\mathrm{AB}^{23}$ & $\mathrm{Cc}\left(20 q+23{ }^{\gtrsim}\right) ; \mathrm{Ni}\left(1 ठ^{\gtrsim}\right), 3+\mathrm{N}$ & $01 / 07 / 19$ & 400 & 1.22 & 105 & 86.1 \\
\hline & $\mathrm{AB}^{23}$ & $\mathrm{Cc}\left(8++11 \delta^{\top}\right) ; \mathrm{Nc}\left(1 \delta^{\top}\right), 2+\mathrm{N}$ & $01 / 07 / 19$ & 200 & 0.38 & 45 & 118.4 \\
\hline & $\mathrm{ID}^{3}$ & $\mathrm{Cc}\left(3 q+2 \jmath^{\prime}\right)$ & $01 / 07 / 19$ & 238 & 0.91 & 5 & 5.5 \\
\hline & $\mathrm{SC}^{1}$ & 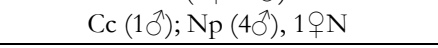 & $01 / 07 / 19$ & 104 & 0.50 & 7 & 14.0 \\
\hline Malvaceae & & & & & & & \\
\hline Theobroma cacao $\mathrm{L}$. & $\mathrm{Sa}^{* *}$ & - & $10 / 26 / 18$ & 10 & 3.44 & 0 & 0 \\
\hline Moraceae & & & & & & & \\
\hline Ficus sp. & $\mathrm{ID}^{20}$ & - & $10 / 26 / 18$ & 61 & 0.30 & 0 & 0 \\
\hline Morus nigra $\mathrm{L}$. & $\mathrm{AB}^{7}$ & - & $10 / 23 / 18$ & 46 & 0.04 & 2 & 50.0 \\
\hline Myrtaceae & & & & & & & \\
\hline Eugenia uniflora $\mathrm{L}$. & $\mathrm{SC}^{1}$ & - & $10 / 22 / 18$ & 176 & 0.70 & 0 & 0 \\
\hline & $\mathrm{AB}^{7}$ & $\operatorname{Af}(3$ 우 $)$ & $10 / 23 / 18$ & 14 & 0.02 & 3 & 150.0 \\
\hline Psidium guajava $\mathrm{L}$. & $\mathrm{CSF}^{* *}$ & $\mathrm{Cc}\left(19 q+29 \jmath^{\circ}\right)$ & $10 / 23 / 18$ & 16 & 2.39 & 74 & 30.9 \\
\hline & $\mathrm{PR}^{11}$ & - & $10 / 24 / 18$ & $32^{\mathrm{d}}$ & 5.50 & 0 & 0 \\
\hline & $\mathrm{PR}^{11}$ & - & $10 / 24 / 18$ & $10^{\mathrm{d}}$ & 1.58 & 0 & 0 \\
\hline & $\mathrm{PR}^{12}$ & - & $10 / 24 / 18$ & $12^{\mathrm{d}}$ & 1.54 & 0 & 0 \\
\hline & $\mathrm{CSF}^{13}$ & - & $10 / 24 / 18$ & $16^{\mathrm{e}}$ & 1.20 & 0 & 0 \\
\hline & $\mathrm{CSF}^{14}$ & - & $10 / 24 / 18$ & $10^{\mathrm{d}}$ & 1.54 & 0 & 0 \\
\hline & $\mathrm{CSF}^{16}$ & - & $10 / 24 / 18$ & $12^{\mathrm{e}}$ & 1.20 & 0 & 0 \\
\hline & $\mathrm{CSF}^{* *}$ & Cc $(1 \widehat{\jmath}) ;$ As (8+), $9 \widehat{\partial A}$ & $01 / 08 / 19$ & 18 & 1.86 & 18 & 9.7 \\
\hline & $\mathrm{CSF}^{* *}$ & $\mathrm{Cc}\left(17 q+13 \jmath^{-1}\right)$ & $01 / 08 / 19$ & 14 & 1.58 & 42 & 26.6 \\
\hline Oxalidaceae & & & & & & & \\
\hline Averrboa carambola $\mathrm{L}$. & $\mathrm{SC}^{1}$ & Ao (7우), $8{ }^{\lambda} \mathrm{A}$ & $10 / 22 / 18$ & 2 & 0.16 & 16 & 100.0 \\
\hline & $\mathrm{AB}^{8}$ & Ao (13q), 12 §ิA, Da (2) & $10 / 23 / 18$ & 19 & 0.70 & 39 & 55.7 \\
\hline & $\mathrm{AB}^{7}$ & $\mathrm{Cc}(9+9+9 \widehat{)}) ;$ Ao $(25 \%), 22 \widehat{\jmath} \mathrm{A}$ & $01 / 07 / 19$ & 18 & 1.02 & 114 & 111.7 \\
\hline & $\mathrm{ID}^{25}$ & 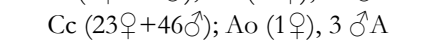 & $01 / 07 / 19$ & 12 & 0.88 & 150 & 170.5 \\
\hline & $\mathrm{SC}^{1}$ & $\mathrm{Cc}\left(1++1{ }^{\top}\right) ;$ Ao $(31$ \% $), 29 \widehat{A}, \mathrm{Da}(5)$ & $01 / 07 / 19$ & 34 & 0.60 & 110 & 183.3 \\
\hline
\end{tabular}




\section{Barreto et al.}

Table 3. Flies (Tephritidae and Lonchaeidae) and their hosts in municipalities of the state of Sergipe. Continuation...

Tabela 3. Moscas (Tephritidae e Lonchaeidae) e respectivos hospedeiros em municípios do estado de Sergipe. Continuação...

\begin{tabular}{|c|c|c|c|c|c|c|c|}
\hline $\begin{array}{l}\text { Families* } \\
\text { Species }\end{array}$ & Spots $\#$ & Flies / Parasitoids & Dates & $\mathrm{F}(\mathrm{n})$ & $\mathrm{M}(\mathrm{kg})$ & $\mathrm{PP}(\mathrm{n})$ & $\mathrm{I}(\mathrm{PP} / \mathrm{kg})$ \\
\hline \multicolumn{8}{|l|}{ Passifloraceae } \\
\hline \multirow[t]{5}{*}{ Passiflora edulis Sims } & $\mathrm{SC}^{2}$ & - & $10 / 22 / 18$ & 3 & 0.54 & 0 & 0 \\
\hline & $\mathrm{ID}^{6}$ & - & $10 / 22 / 18$ & 26 & 1.70 & 0 & 0 \\
\hline & $\mathrm{AB}^{7}$ & - & $10 / 23 / 18$ & 6 & 0.64 & 0 & 0 \\
\hline & $\mathrm{L}^{* *}$ & - & $10 / 23 / 18$ & 10 & 1.50 & 0 & 0 \\
\hline & $\mathrm{CSF}^{17}$ & - & $10 / 24 / 18$ & 11 & 2.90 & 0 & 0 \\
\hline \multicolumn{8}{|l|}{ Rutaceae } \\
\hline Citrus sinensis (L.) Osbeck & $\mathrm{ID}^{5}$ & - & $10 / 22 / 18$ & 27 & 5.86 & 0 & 0 \\
\hline \multirow[t]{2}{*}{ Citrus reticulata Blanco } & $\mathrm{AB}^{9}$ & $\mathrm{Cc}(12++8 \hat{)}) ; \mathrm{Nc}(1 \widehat{\jmath})$ & $10 / 23 / 18$ & 4 & 0.52 & 23 & 44.2 \\
\hline & $\mathrm{TB}^{* *}$ & - & $01 / 08 / 19$ & 1 & 0.18 & 0 & 0 \\
\hline \multicolumn{8}{|l|}{ Sapotaceae } \\
\hline \multirow[t]{4}{*}{ Manilkara rapota (L.) P. Royen } & $\mathrm{SC}^{1}$ & - & $10 / 22 / 18$ & 6 & 0.96 & 0 & 0 \\
\hline & $I^{* *}$ & - & $10 / 23 / 18$ & 10 & 1.06 & 0 & 0 \\
\hline & $\mathrm{Sa}^{* *}$ & - & $01 / 08 / 19$ & 11 & 1.38 & 0 & 0 \\
\hline & SC** & - & $01 / 10 / 19$ & 10 & 0.55 & 0 & 0 \\
\hline \multicolumn{8}{|l|}{ Solanaceae } \\
\hline \multirow[t]{2}{*}{ Capsicum sp. } & $\mathrm{CSF}^{4}$ & - & $10 / 22 / 18$ & 8 & 0.08 & 0 & 0 \\
\hline & $\mathrm{AB}^{7}$ & - & $10 / 23 / 18$ & 4 & 0.06 & 0 & 0 \\
\hline Capsicum frutescens $\mathrm{L}$. & $\mathrm{CSF}^{17}$ & - & $10 / 24 / 18$ & 185 & 0.25 & 0 & 0 \\
\hline Verbenaceae & $A B^{8}$ & & $10 / 23 / 18$ & 679 & 028 & 0 & 0 \\
\hline
\end{tabular}

*The scientific names are in accordance with The Plant List (2013) and Flora do Brasil (2020). \# Municipalities: A: Aracaju, AB: Areia Branca, BC: Barra dos Coqueiros, CSF: Canindé de São Francisco, I: Itabaiana, ID: Itaporanga D’Ajuda, L: Lagarto, PR: Poço Redondo, R: Ribeirópolis, Sa: Salgado, SC: São Cristóvão, S: Siriri, TB: Tobias Barreto. For coordinates of sampling sites (from 1 to 29 ), see Table 1. **Fruits purchased at farmers markets. ${ }^{a}$ Manga Rosa; ${ }^{b}$ Manga Comum; ${ }^{\mathrm{c}}$ Manga Espada; dVariety Paluma; ${ }^{\mathrm{G}} \mathrm{Goiabeira}$ espontânea. $\mathrm{F}=$ number of fruits; $\mathrm{M}=$ mass; $\mathrm{PP}=$ puparia; $\mathrm{I}=$ infestation; Af: Anastrepha fraterculus; Ao: Anastrepha

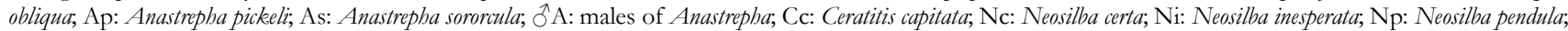
QN : Neosilba sp.; Da: Doryctobracon areolatus. F = Number of fruits; $\mathrm{M}=$ mass.

Table 4. Flies (Tephritidae and Lonchaeidae) caught in McPhail traps in the state of Sergipe.

Tabela 4. Moscas (Tephritidae e Lonchaeidae) capturadas em armadilhas tipo McPhail no estado de Sergipe.

\begin{tabular}{|c|c|c|c|c|}
\hline Municipality & Period & Latitude S & Longitude $\mathrm{W}$ & Species* \\
\hline Aracaju & $\operatorname{Jan} / 2019$ & $11^{\circ} 04^{\prime} 19.3^{\prime \prime}$ & $37^{\circ} 07^{\prime} 20.3^{\prime \prime}$ & 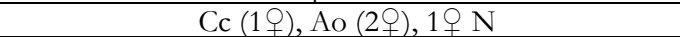 \\
\hline \multirow{2}{*}{ Areia Branca } & Out/2018 & $10^{\circ} 45^{\prime} 41.6^{\prime \prime}$ & $37^{\circ} 18^{\prime} 05.4^{\prime \prime}$ & 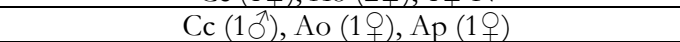 \\
\hline & $\operatorname{Jan} / 2019$ & $10^{\circ} 45^{\prime} 41.6^{\prime \prime}$ & $37^{\circ} 18^{\prime} 05.4^{\prime \prime}$ & $\mathrm{Cc}(11 \Phi+3 \hat{\circ}), \mathrm{Ao}(10 \Phi), 1 \widehat{O A}$ \\
\hline \multirow{2}{*}{ Itabaiana } & $\operatorname{Tan} / 2019$ & $10^{\circ} 44^{\prime} 17.3^{\prime \prime}$ & $37^{\circ} 24^{\prime} 22.2^{\prime \prime}$ & $\operatorname{Cc}(39)$ \\
\hline & $\operatorname{Jan} / 2019$ & $10^{\circ} 43^{\prime} 32.5^{\prime \prime}$ & $37^{\circ} 23^{\prime} 48.8^{\prime \prime}$ & Cc $(19)$ \\
\hline \multirow{3}{*}{ Itaporanga D'Ajuda } & Out/2018 & $10^{\circ} 59^{\prime} 45.2^{\prime \prime}$ & $37^{\circ} 20^{\prime} 0.03^{\prime \prime}$ & Cc $\left(10^{3}\right)$ \\
\hline & $\operatorname{Jan} / 2019$ & $10^{\circ} 59^{\prime} 50.0^{\prime \prime}$ & $37^{\circ} 19^{\prime} 52.0^{\prime \prime}$ & Cc $(39), \operatorname{Ao}(29)$ \\
\hline & $\operatorname{Jan} / 2019$ & $10^{\circ} 59^{\prime} 45.2^{\prime \prime}$ & $37^{\circ} 20^{\prime} 0.03^{\prime \prime}$ & Cc (8ㅇ), Ao (9ㅇ), Ap (69), Af (4ㅇ), As (29), $9 \bigcirc^{\wedge} \mathrm{A}$ \\
\hline \multirow{2}{*}{ São Cristóvão } & $\operatorname{Jan} / 2019$ & $10^{\circ} 58^{\prime} 18.0^{\prime \prime}$ & $37^{\circ} 10^{\prime} 43.1^{\prime \prime}$ & Cc $\left(149+4 \delta^{\lambda}\right)$, Ao $(1829), \operatorname{Af}(29)$, As $(19), 37 \oint^{\lambda} \mathrm{A}$ \\
\hline & Jan/2019 & $10^{\circ} 58^{\prime} 18.0^{\prime \prime}$ & $37^{\circ} 10^{\prime} 43.1 "$ & Cc (19), Ao (86ㅇ), Af (3ㅇ), Aps (3), As (19), $22 \bigcirc^{\lambda} \mathrm{A}$ \\
\hline
\end{tabular}

Table 5. Flies (Tephritidae and Lonchaeidae) reported for the state of Sergipe, Brazil.

Tabela 5. Moscas (Tephritidae e Lonchaeidae) reportadas para o estado de Sergipe, Brasil.

\begin{tabular}{ll}
\hline \multicolumn{1}{c}{ Species } & Municipalities \\
\hline Anastrepha fraterculus (Wiedemann) & $\mathrm{A}, \mathrm{AB}, \mathrm{ID}$ \\
Anastrepha obliqua (Macquart) & $\mathrm{A}, \mathrm{AB}, \mathrm{CSF}, \mathrm{ID}, \mathrm{SC}, \mathrm{S}$ \\
Anastrepha sororcula Zucchi & $\mathrm{CSF}, \mathrm{ID}, \mathrm{SC}$ \\
Anastrepha pickeli Lima & $\mathrm{AB}, \mathrm{ID}, \mathrm{SC}, \mathrm{S}$ \\
Anastrepha pseudoparallela (Loew) & $\mathrm{SC}$ \\
Ceratitis capitata (Wiedemann) & $\mathrm{A}, \mathrm{AB}, \mathrm{CSF}, \mathrm{I}, \mathrm{ID}, \mathrm{SC}$ \\
Neosilba certa (Walker) & $\mathrm{AB}$ \\
Neosilba inesperata Strikis \& Prado & $\mathrm{AB}, \mathrm{CSF}$ \\
Neosilba pendula (Bezzi) & $\mathrm{SC}$ \\
\hline
\end{tabular}

Municipalities: A: Aracaju, AB: Areia Branca, CSF: Canindé de São Francisco, I: Itabaiana, ID: Itaporanga D’Ajuda, SC: São Cristóvão, S: Siriri.

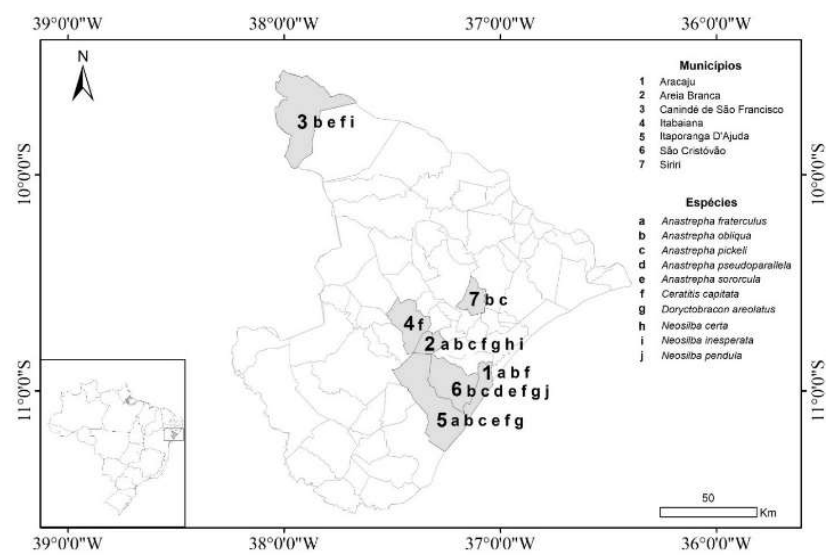

Figure 1. Geographic distribution of species of frugivorous flies and parasitoid in the state of Sergipe.

Figura 1. Distribuição geográfica das espécies de moscas frugívoras e parasitoide no estado de Sergipe. 


\section{DISCUSSION}

Since 1980, A. fraterculus, obtained from an occasional sample of guava in São Cristóvão, was the only record of fruit flies for the state of Sergipe (MALAVASI et al., 1980). After almost four decades, this paper presents the results of the first survey on Tephritidae and Lonchaeidae in the state of Sergipe. Consequently, with the exception of $A$. fraterculus record in P. guajava (MALAVASI et al., 1980), all other results presented in this paper (flies, host plants and parasitoid) are new for the state of Sergipe. Therefore, four species of Anastrepha, Ceratitis capitata, three of Neosilba, one of parasitoid braconid and nine of hosts are new records for the state (Table 5 and Figure 1).

Anastrepha obliqua and C. capitata infested the largest number of hosts (Table 3). Ceratitis capitata was obtained mainly in exotic hosts, and guava was the only native host attacked by this species. This data may be indicative of recent presence of $C$. capitata in Sergipe and, therefore, has not yet adapted to native hosts. On the other hand, the species occurs in the neighboring states of Alagoas, Bahia and Pernambuco (ZUCCHI; MORAES, 2012). Anastrepha obliqua and C. capitata are among the most polyphagous flies in Brazil, as they are associated to 50 and 94 plant species, respectively (ZUCCHI; MORAES, 2008; 2012). Despite passion fruit sampling, preferred host of $A$. pseudoparallela, in five municipalities, no specimens of this species were obtained from this host. In the traps, only 10 specimens of $A$. pseudoparallela were collected (Table 4).

The municipalities sampled are located in the costal, transitional semi-arid and hinterland regions. However, more flies were collected in the coastal and more urbanized area of Sergipe (Aracaju and São Cristóvão) in comparison to the other regions. Of the 13 municipalities sampled, in only five of them dipterans were captured in traps (Table 4).

The sampling was conducted in an area with several fruit trees and close to a fragment of the Atlantic Rainforest, a favorable factor for richness and diversity (ARAUJO et al., 2008; DUTRA et al., 2009). Several factors are related to diversity and abundance of fruit flies; however, climate generally regulates the development of native hosts and natural antagonism between species (ALUJA et al., 1997). The occurrence of fruit fly species depends on preference of hosts and is affected by the landscape, that is, the forestgrowing system (MONTEIRO et al., 2018). According to these authors, in the metropolitan region of Curitiba (Paraná State - Brazil), many orchards are near or surrounded by fragments of the Atlantic Forest, which leads to interspecific competition. Although many native and cultivated plant species are not considered to be the main hosts of fruit flies, these plants play a crucial role in the multiplication of flies and their natural enemies. The Atlantic Forest has one of the largest diversities of plant and animal species (MARTINI et al., 2007) and contains native and exotic host plants for fruit flies.

Considering that the three species of lance flies have infested marketable fruits in some municipalities, studies should be intensified to estimate losses in acerola and tangerine caused by these species. Acerola is infested by $N$. certa and N. inesperata in the state of São Paulo (RAGA et al., 2015) and by N. pendula in the state of Bahia (LEITE et al., 2016). Neosilba certa was also recorded in tangerine in the state of Rio de Janeiro (SOUZA et al., 2008).
Only one species of parasitoid braconid (D. areolatus) was obtained from fruit samplings (A. obliqua in $A$. carambola and $S$. dulcis). This parasitoid is the most common and widely distributed in Brazil (CANAL; ZUCCHI, 2000, MARINHO et al., 2018), also in South America (OVRUSKI et al., 2000). Parasitism rates were low, compared to other surveys conducted in Brazil. Natural parasitism varies in the different fruit producing regions, since it is influenced by several factors (OVRUSKI et al., 2000).

Considering the socioeconomic importance of fruit growing for the state of Sergipe, further surveys on dipterans are recommended, regarding economic losses, their host plants, geographic distribution and time of occurrence. This knowledge is crucial for the adoption of management strategies for effective control of these agricultural pests and reduction of damages to the fruit production. Studies on parasitoids, which act on the natural population regulation of fruit flies, are also recommended.

\section{CONCLUSIONS}

In addition to Anastrepha species and Ceratitis capitata, Neosilba species infest marketable fruits in the state of Sergipe.

\section{ACKNOWLEDGEMENTS}

The authors thank the rural producers who graciously allowed collections in their properties. We give thanks to the National Council for Scientific and Technological Development - CNPq for the Research Productivity Fellowship granted to Ricardo Adaime and Roberto Antonio Zucchi and for the Postdoctoral Fellowship granted to the first author. We also thank T. Zamadei for the map elaboration.

\section{REFERENCES}

ADAIME, R.; SOUSA, M. S. M.; JESUS-BARROS, C. R.; DEUS, E. G.; PEREIRA, J. F.; STRIKIS, P. C.; SOUZA-FILHO, M. F. Frugivorous flies (Diptera: Tephritidae, Lonchaeidae), their host plants, and associated parasitoids in the extreme north of Amapá State, Brazil. The Florida Entomologist, Lutz, v. 100, n. 2 , p. 316-324, 2017. DOI: https://dx.doi.org/10.1653/024.100.0229

ALUJA, M.; JIMÉNEZ, A.; CAMINO, M.; PIÑERO, J.; ALDANA, L.; CASTREJÓN, V.; VALDÉS, M. E. Habitat Manipulation to Reduce Papaya Fruit Fly (Diptera: Tephritidae) Damage: Orchard Design, Use of Trap Crops and Border Trapping. Journal of Economic Entomology, Lanham, v. 90, n. 6, p. 1567 1576, $1987 . \quad$ DOI: https://dx.doi.org/10.1093/jee/90.6.1567

ARAUJO, E. L.; SILVA, R. K.; GUIMARÃES, J. A.; SILVA, J. G.; BITTENCOURT, M. A. L. Levantamento e flutuação populacional de moscas-dasfrutas (Diptera: Tephritidae) em goiaba Psidium guajava L., no município de Russas (CE). Revista Caatinga, Mossoró, v. 21, n.1, p.138-146, 2008.

BRASIL, 2018. Instrução Normativa ${ }^{\circ} 38$, de 1 de outubro de 2018. Diário Oficial [da] União, Brasília, DF, 2 out. 2018, Seção 1, p. 14. 
CANAL, N. A.; ZUCCHI, R. A. Parasitóides - Braconidae. In: Malavasi, A.; ZUCCHI, R. A. (Ed.). Moscas-dasfrutas de Importância Econômica no Brasil. Ribeirão Preto, Holos, 2000. p. 119-126.

DUTRA, V. S.; SANTOS, M. S.; SOUZA FILHO, Z. A.; ARAUJO, E. L.; SILVA, J. G. Faunistic analysis of Anastrepha spp. (Diptera: Tephritidae) on a guava orchard under organic management in the municipality of Una, Bahia, Brasil. Neotropical Entomology, Londrina, v. 38, n. 1, p. 133-138, 2009. DOI: http://dx.doi.org/10.1590/S1519-566X2009000100015

FLORA DO BRASIL - em construção. Jardim Botânico do Rio de Janeiro, 2020. Disponível em: $<$ http://floradobrasil.jbrj.gov.br/ > . Acesso em: 08 mai. 2020

LEITE, S. A.; CASTELLANI, M. A.; RIBEIRO, A. E. L.; COSTA, D. R. da; BITTENCOURT, M. A. L.; MOREIRA, A. A. Fruit flies and their parasitoids in the fruit growing region of Livramento de Nossa Senhora, Bahia, with records of unprecedented interactions. Revista Brasileira de Fruticultura, Jaboticabal, v. 39, n. 4: 2016. (e-592) DOI: https://dx.doi.org/10.1590/010029452017592

MALAVASI, A.; MORGANTE, J. S.; ZUCCHI, R. A. Biologia de moscas-das-frutas (Diptera: Tephritidae) I. Lista de hospedeiros e ocorrência. Revista Brasileira de Biologia, Rio de Janeiro, v. 40, n. 1, p. 9-16, 1980.

MALAVASI, A.; ZUCCHI, R. A. Moscas-das-frutas de importância econômica no Brasil: conhecimento básico e aplicado. Ribeirão Preto: Holos, 2000. 324 p.

MARINHO, C. F.; COSTA, V. A.; ZUCCHI, R. A. Annotated checklist and illustrated key to braconid parasitoids (Hymenoptera, Braconidae) of economically important fruit flies (Diptera, Tephritidae) in Brazil. Zootaxa, Auckland, v. 4527, n. 1, p. 21-36, 2018. DOI: https://dx.doi.org/10.11646/zootaxa.4527.1.2

MARTINI, A. M. Z.; FIASCHI, P.; AMORIM, A. M.; PAIXÃO, J. L. A hot-point within a hot-spot: a high diversity site in Brazil's Atlantic Forest. Biodiversity and Conservation, London, v. 16, n. 11, p. 3111-3128, 2007. DOI: https://dx.doi.org/10.1007/s10531-007-9166-6

MONTEIRO, L. B.; TOMBA, J. A. S.; NISHIMURA, G.; MONTEIRO, R. S.; FOELKEL, E.; LAVIGNE, C. Faunistic analyses of fruit fly species (Diptera: Tephritidae) in orchards surrounded by Atlantic Forest fragments in the metropolitan region of Curitiba, Paraná state, Brazil. Brazilian Journal of Biology, São Carlos, v. 79 , n. 3, p. 395-403, 2018. DOI: http://dx.doi.org/10.1590/1519-6984.178458

OVRUSKI, S. M.; ALUJA, M.; SIVINSKI, J.; WHARTON, R. A. Hymenopteran parasitoids on fruit-infesting Tephritidae (Diptera) in Latin America and the Southern
United States: diversity, distribution, taxonomic status and their use in fruit fly biological control. Integrated Pest Management Reviews, v. 5, n. 2, p. 81-107, 2000. DOI: https://dx.doi.org/10.1023/A:1009652431251

RAGA, A.; SOUZA-FILHO, M. F.; STRIKIS, P. C.; MONTES, S. M. N. M. Lance fly (Diptera: Lonchaeidae) host plants in the State of São Paulo, Southeast Brazil. Entomotropica, v. 30, n. 7, p. 57-68, 2015.

SANTOS, M. dos R. Observatório de Sergipe. Disponível em: <http://observatorio.se.gov.br/geografia-ecartografia/mapas-e-cartogramas/>. Acesso em: 31 de janeiro de 2019.

SEMARH/SERGIPE. Sistema de Informações sobre Recursos Hídricos do Estado de Sergipe: Clima. Disponível em: <http://www.semarh.se.gov.br>. Acessado em: 01 de fevereiro de 2019.

SILVA, R. A.; DEUS, E. G.; RAGA, A.; PEREIRA, J. D. B.; SOUZA-FILHO, M. F.; COSTA NETO, S. V. Monitoramento de moscas-das-frutas na Amazônia: amostragem de frutos e uso de armadilhas. In: SILVA, R. A.; LEMOS, W. P.; ZUCCHI, R. A. (Ed.). Moscas-dasfrutas na Amazônia brasileira: diversidade, hospedeiros e inimigos naturais. Macapá: Embrapa Amapá, 2011. p. 33-50.

SOUZA, J. F.; SOUZA, S. A. S.; AGUIAR-MENEZES, E. L.; FERRARA, F. A. A.; NASCIMENTO, S. A.; RODRIGUES, W. C.; CASSINO, P. C. R. Diversity of fruit flies in citrus groves in the municipality of Araruama, RJ. Ciência Rural, Santa Maria, v. 38, p. 518-521, 2008. DOI: 84782008000200035 http://dx.doi.org/10.1590/S0103-

The Plant List. Version 1.1. Published on the Internet; http://www.theplantlist.org/. 2013. Acesso em: 08 mai. 2020

UCHÔA, M. A. Fruit flies (Diptera: Tephritoidea): biology, host plants, natural enemies, and the implications to their natural control. In: LARRAMENDY, M. L.; SOLONESKI, S. (Ed.). Integrated pest management and pest control: current and future tactics. Rijeka: In'Tech, 2012. p. 271-300.

ZUCCHI, R. A. Mosca-do-mediterrâneo, Ceratitis capitata (Wiedemann). In: VILELA, E. F.; ZUCCHI, R. A. (Ed.). Pragas introduzidas no Brasil: insetos e ácaros. Piracicaba: FEALQ, 2015. p. 153-172.

ZUCCHI, R. A.; MORAES, R. C. B. Fruit flies in Brazil Anastrepha species their host plants and parasitoids. 2008. Disponível em: <www.lea.esalq.usp.br/anastrepha/>. Acesso em: 20 mar. 2019.

ZUCCHI, R. A.; MORAES, R.C.B. 2012. Fruit flies in Brazil - Hosts and parasitoids of the Mediterranean fruit fly. Disponível em: <www.lea.esalq.usp.br/ceratitis/>. Acesso em: 07 maio 2020. 